Journal of Mathematics and Informatics

Vol. 17, 2019, 55-65

ISSN: 2349-0632 (P), 2349-0640 (online)

Published 26 July 2019

www.researchmathsci.org

DOI: http://dx.doi.org/10.22457/jmi.147av17a5

Journal of

Mathematics and

Informatics

\title{
Medical Image Fusion Combining Singular Value Decomposition and Mean Gradient
}

\author{
Wang Jing, Zhang Guicang and Su Jinfeng \\ School of Mathematics \& Statistics, Northwest Normal University \\ Gansu Lanzhou, 730000, China \\ Email: 1714986367@qq.com
}

Received 12 June 2019; accepted 9 July 2019

\begin{abstract}
In order to solve the problem that the traditional fusion algorithm can easily cause image distortion and artificial texture in the face of medical images, a medical image fusion algorithm combining singular value decomposition and average gradient is proposed in this paper. The algorithm is divided into the following three main steps: The first step is to decompose the medical images with wavelet. The second step is to select the fusion rule according to the condition: For the high frequency component of the highest decomposition level, singular value decomposition is performed in the neighborhood. Then, the 2- norm of the neighborhood pixel matrix is used as the contrast component to describe the image features, and the mean value of all elements of the middle value difference matrix in the neighborhood is selected as the contrast component for the high frequency and low frequency components of the rest of the decomposition layer. The third step is to reconstruct the image. A large number of experiments and analysis show that this method can effectively solve the problems of traditional algorithms in medical image fusion, to facilitate the doctor's accurate judgments of the patient's condition and to improve the formulation of the treatment plan scientifically.
\end{abstract}

Keywords: Wavelet decomposition; Singular value decomposition; Median difference matrix; Contrast component

AMS Mathematics Subject Classification (2010): 42C40

\section{Introduction}

With the increasing accuracy of image information, image fusion technology has become a hot topic for many scholars. Various image fusion algorithms have a wide range of applications, each with its advantages and disadvantages. In medical clinics, images provided by different medical imaging devices have their own advantages and disadvantages. The image information provided by a single imaging device is limited, which is not conducive to the doctor's correct judgment of the condition. Medical image fusion technology can overcome the limitations and differences brought by a single sensor image, and obtain a comprehensive and accurate description of the scene to improve the clarity and recognition of the image. Therefore, the application of medical image fusion throughout the clinical work, not only widely used in the diagnosis of disease, but also plays an important role in surgery and radiation therapy [1]. 


\section{Medical Image Fusion Combining Singular Value Decomposition and Mean Gradient}

Traditional medical image fusion methods mainly include weighted average method, Laplace pyramid transform, wavelet transform [13] and multi-resolution analysis based fusion algorithm. For example, in document [2], a weighted fusion method based on multiple directions including horizontal direction is introduced to fuse source image and Laplace pyramid fusion image according to the weighted fusion rule, which makes it easy to add the noise of artificial texture and fused image. Literature [3] proposed that the low-frequency information should be fused according to the proposed improved weighted average method. Although the spatial resolution of the image can be improved, it is difficult to select the uncertainty of the threshold. In reference [4], it is proposed that high-frequency [19] and low-frequency components can be obtained by using wavelet transform, and then the principle of maximizing directional contrast and average gradient is applied in the neighborhood, and the corresponding fusion rules are selected to obtain the fused image which is easy to cause image distortion. In reference [5], different component matrices are obtained after singular value decomposition of source image. According to the properties of matrix, they are defined as smoothing component and detail component respectively, and then the singular value decomposition is continued. This is relatively simple for complex image processing, but the definition of smoothing component and detail component after decomposition needs further verification. Although these algorithms can highlight the details of the fused image, they can not reflect the boundary information of the image very well. Moreover, medical image fusion algorithms do not involve the properties of low rank matrix formed by image fusion, which usually leads to image fusion distortion and image information loss[6-8,18].

To sum up the above problems, this paper uses the singultion level, the singular value decomposition is performed in the neighborhood. Then the 2-norm of the neighborhood pixel matrix is used as the contrast component to describe the image features. The mean of all elements of the median difference matrix in the neighborhood is selected as the contrast component for the details and approximation of the other decomposition levels. Finally, the image is reconstructed. The experimental results and analysis show that the proposed algorithm can effectively solve the problems existing in the traditional algorithm in medical image fusion, and help doctors make accurate judgments and design scientific treatment plans for patients in medical diagnosis.

\section{Singular value decomposition and contrastive fusion algorithms}

\subsection{Singular value decomposition (SVD) theory}

Singular value decomposition (SVD) is an orthogonal transformation with the best correlation. For any row-column linear correlation matrix, a diagonal matrix is obtained by multiplying each unitary matrix. The number of singular values can reflect the number of row (column) vectors of the original matrix. For a matrix with large amount of data, SVD can remove the redundant information in the image, and only concentrate the information on a few singular values [5].

According to literature [9], the following lemma can be obtained:

Theorem 2.1. [9](SVD decomposition)Let $A$ be an $m \times n$ matrix, and $\operatorname{rank}(A)=r$ then there are $m$-order unitary matrices $V$ and $n$-order unitary matrices $U$ such that

$$
U^{H} A V=\left[\begin{array}{ll}
\Sigma & 0 \\
0 & 0
\end{array}\right]
$$


Wang Jing, Zhang Guicang and Su Jinfeng

Matrix can $A$ be decomposed into the product of three matrices.

$$
A=U \Sigma V^{H}
$$

where $\sum=\operatorname{diag}\left(\sigma_{1}, \ldots, \sigma_{r}\right)$, and $\sigma_{1} \geq \cdots \geq \sigma_{r}>0$.

Theorem 2.3. [9] Assume $A=\left(a_{i j}\right) \in C^{m \times n}$. Then there is

$$
\|A\|_{2}=\left(\lambda_{\max }\left(A^{H} A\right)\right)^{\frac{1}{2}}
$$

\subsection{Wavelet transform image fusion}

The advantage of applying wavelet transform to medical image fusion is that it can decompose the image into different frequency domains, and use different selection rules in different frequency domains to obtain accurate multi-resolution decomposition of the composite image, thereby preserving the source image in the composite image. Significant features in different frequency domains. The main steps are as follows ${ }^{[1]}$.

(1) Geometrically accurate registration of multi-source images;

(2) Choose appropriate wavelet bases and decomposition layers, and decompose the original image with wavelet transform to get their respective details and smoothing components.

(3) The fusion rules of wavelet coefficients are selected according to the specific needs.

(4) Fusion image is obtained by inverse transformation.

\subsection{Singular value decomposition based on wavelet transform}

Singular value decomposition (SVD) is mostly used in multi-resolution image fusion. The SVD of image matrix is decomposed into uncorrelated smoothing and detail components, and the smoothing components are decomposed and processed at multiple levels. Although it is simple and convenient for complex image processing, the definition of approximation and detail after decomposition still needs further verification. In this paper, singular value decomposition (SVD) algorithm based on wavelet transform is introduced. On the basis of wavelet transform, high-frequency components and low-frequency components can be obtained.

Taking the fusion of two images $A$ and $B$ as an example, The fused image is $F$.Two-dimensional image is decomposed by $N$ layer wavelet transform, Ultimately, there are $(3 N+1)$ different bands, It contains $3 N$ high frequency components and one low frequency component. So we get the following four sets of coefficients $\left[c A_{j}, c D_{j}^{H}, c D_{j}^{V}, c D_{j}^{D}\right]$. among them, $c A_{j}$ is the approximation coefficient of layer $j$; $c D_{j}^{H}$ is the horizontal component of the detail parameter of layer $j ; c D_{j}^{V}$ is the vertical component of the detail parameters of layer $j ; c D_{j}^{D}$ is the diagonal component of the detail parameters of Layer $j$.

Horizontal component of the highest decomposition layer $c D_{N}^{H}$ 、 Vertical component $c D_{N}^{V}$ and Diagonal component $c D_{N}^{D}$ Singular value decomposition based on formula (2) is performed in the neighborhood centered on $(i, j)$ pixels, The singular value 
Medical Image Fusion Combining Singular Value Decomposition and Mean Gradient decomposition matrix in the neighborhood is obtained.

$$
c D_{N}^{i}=V_{i}\left[\begin{array}{cc}
\sum & 0 \\
0 & 0
\end{array}\right] U^{H}
$$

Among them, $V_{i}$ and $U$ are unitary matrices, $N$ are the highest decomposition levels, $\sum=\operatorname{diag}\left(\sigma_{1}, \ldots, \sigma_{k}\right)$, and $\sigma_{1} \geq \cdots \geq \sigma_{r}>0$ is the singular value of $c D_{N}^{i}, i=H, V, D$ denotes the horizontal, vertical and diagonal components.

Formula (3) shows that the square of the 2-norm of the matrix represents the maximum singular value of the matrix, and the 2-norm of the neighborhood pixel matrix can be used as the contrast component to describe the image characteristics:

$$
\left\|c D_{N}^{i}(i, j)\right\|_{2}^{2}=\lambda_{\max }\left(\left(c D_{N}^{i}(i, j)\right)^{H}\left(c D_{N}^{i}(i, j)\right)\right)=\sigma_{N \max }^{i}(i, j)
$$

Among them, $\sigma_{N \max }^{i}(i, j)$ is the maximum singular value of the pixel matrix in the neighborhood centered on $(i, j)$.

Then, the whole high frequency coefficient matrix is decomposed by singular value decomposition. The ratio of the maximum singular value in the corresponding neighbourhood to the maximum singular value of the coefficient matrix is taken, and the pixel points of the source image corresponding to the maximum value are compared.

$$
\left\|c D_{N}^{i}\right\|_{2}^{2}=\lambda_{\max }\left(\left(c D_{N}^{i}\right)^{H}\left(c D_{N}^{i}\right)\right)=\sigma_{N \max }^{i}
$$

Among them, $\sigma_{N \max }^{i}$ is the maximum singular value of the coefficient matrix.

$$
\sigma_{N}^{i}=\frac{\sigma_{N \max }^{i}(i, j)}{\sigma_{N \max }^{i}}
$$

Among them, $\sigma_{N}^{i}$ is the maximum singular value contrast value in the neighborhood.

The fusion rules are as follows:

$$
\begin{gathered}
D_{N, F}^{H}(i, j)=\left\{\begin{array}{l}
c D_{N, A}^{H}(i, j), \sigma_{N, A}^{H}(i, j) \geq \sigma_{N, B}^{H}(i, j) \\
c D_{N, B}^{H}(i, j), \sigma_{N, A}^{H}(i, j)<\sigma_{N, B}^{H}(i, j)
\end{array}\right. \\
D_{N, F}^{V}(i, j)=\left\{\begin{array}{l}
c D_{N, A}^{V}(i, j), \sigma_{N, A}^{V}(i, j) \geq \sigma_{N, B}^{V}(i, j) \\
c D_{N, B}^{V}(i, j), \sigma_{N, A}^{V}(i, j)<\sigma_{N, B}^{V}(i, j)
\end{array}\right. \\
D_{N, F}^{D}(i, j)=\left\{\begin{array}{l}
c D_{N, A}^{D}(i, j), \sigma_{N, A}^{D}(i, j) \geq \sigma_{N, B}^{D}(i, j) \\
c D_{N, B}^{D}(i, j), \sigma_{N, A}^{D}(i, j)<\sigma_{N, B}^{D}(i, j)
\end{array}\right.
\end{gathered}
$$

Among them $D_{N}^{l}(i, j)(l=H, V, D)$ represents the level of the highest decomposition level of the fused image $F$, respectively. The coefficients of the vertical and diagonal components at point $(i, j)$.

\subsection{Singular value contrast fusion based on wavelet transform} The steps of singular value contrast fusion are as follows:

(1) Determining the size of neighborhood;

(2) For a high frequency coefficient matrix in the highest decomposition level, the 
Wang Jing, Zhang Guicang and Su Jinfeng

maximum singular value of the pixel matrix in the neighborhood centered on $(i, j)$ is obtained;

(3) The maximum singular value of a high frequency coefficient matrix corresponding to the highest decomposition level is obtained.

(4) The ratio of the maximum singular value in the corresponding neighborhood to the maximum singular value of the coefficient matrix is taken to compare the pixels of the source image corresponding to the maximum.

\section{Average gradient meaning principle}

\subsection{Definition of average gradient}

Average gradient refers to the obvious difference of gray level near the edge points or shadow lines of an image, that is, the gray change rate. It reflects the rate at which minute details of an image change and represents the degree of pixel sharpness of the image.

Its definition is as follows (9):

$$
\bar{g}=\frac{1}{M \times N} \sum_{i=0}^{M-1} \sum_{j=0}^{N-1} \sqrt{\frac{\Delta I_{x}^{2}+\Delta I_{y}^{2}}{2}}
$$

Among them, $\Delta I_{x}$ and $\Delta I_{y}$ are the difference in $x$ direction and $y$ direction of $M \times N$ size images respectively.

\subsection{Average gradient meaning method}

The bigger the average gradient of the image, the better the clarity of the representation. Firstly, the coefficients matrix can be obtained by $N$-layer wavelet decomposition of source image $A$ and $B$,For the low frequency coefficient matrix of the highest decomposition layer and all coefficient matrices of other layers, the average gradient of the matrix is obtained by using formula (9) in the $M \times N$ neighborhood centered on $(i, j)$.

$$
\bar{g}(i, j)=\frac{1}{M \times N} \sum_{i=0}^{M-1} \sum_{j=0}^{N-1} \sqrt{\frac{\Delta I_{x}^{2}+\Delta I_{y}^{2}}{2}}
$$

Secondly, the following formula is defined (11):

$$
T=\frac{\Delta I_{x}^{2}+\Delta I_{y}^{2}}{2}
$$

Then, the mean values of all elements of the median difference matrix in the neighborhood of the image are obtained by using formula (12), that is, the mean values of all elements of the median difference matrix in the neighborhood of the image

$$
g(i, j)=\frac{1}{M \times N} \sum_{i=0}^{M} \sum_{j=0}^{N} T(i, j)
$$

Among them, $M$ and $N$ are the size of matrix $T$.

Finally, the fusion rule defines the following formulas (13) and (14):

$$
G(i, j)=\frac{g(i, j)}{g(i, j)}
$$

then

$$
C_{n, F}(i, j)=\left\{\begin{array}{l}
C_{n, A}(i, j), G_{n, A}(i, j) \geq G_{n, B}(i, j) \\
C_{n, B}(i, j), G_{n, A}(i, j)<G_{n, B}(i, j)
\end{array}\right.
$$


Medical Image Fusion Combining Singular Value Decomposition and Mean Gradient

\subsection{Fusion steps of mean gradient averaging algorithm based on Wavelet transform} The average gradient averaging fusion steps are as follows:

(1) Determining the size of neighborhood;

(2) The average gradient in the neighborhood centered on $(i, j)$ is obtained for a frequency domain coefficient matrix of the highest decomposition layer;

(3) The mean values of all elements of the corresponding $(i, j)$ centered neighborhood difference matrix are obtained for a frequency domain coefficient matrix corresponding to the highest decomposition level;

(4) The ratio of the mean of all elements to the mean gradient of the median difference matrix in the corresponding neighborhood is taken to compare the pixels of the source image corresponding to the maximum.

\section{Combining singular value decomposition and average gradient fusion algorithms 4.1. Wavelet decomposition}

Two source images $A 、 B$ are decomposed into $3 N$ high frequency components and one low frequency component by $N$-layer wavelet decomposition.

\subsection{Contrast and mean gradient of singular value decomposition}

Firstly, the maximum singular value is obtained by singular value decomposition of the high-frequency components of the highest decomposition level, and the ratio of the maximum singular value in the corresponding neighborhood to the maximum singular value of the coefficient matrix is taken to compare the pixels of the source image corresponding to the largest one. Then, the mean gradient in the neighborhood is calculated for the low-frequency component of the highest decomposition layer and the high-frequency component of the other decomposition layers, and the mean values of all elements of the median difference matrix in the corresponding neighborhood are obtained. Finally, the ratio of the mean of all elements to the mean gradient of the median difference matrix in the neighborhood is obtained, and the pixels of the source image corresponding to the largest one are compared.

\subsection{Image reconstruction}

After determining the high-frequency and low-frequency components of each layer of the fused image $F$, the fused image $F$ is reconstructed by inverse wavelet transform.

\section{Experimental results and analysis}

In order to verify the role of singular value decomposition and mean gradient averaging in medical image fusion, two CT and MRT images of $256 \times 256$ size were selected for fusion experiments. Fig. 1 shows the experimental results of the wavelet fusion algorithm in different cases.

In the experiment, the wavelet decomposition level of the wavelet fusion algorithm is 3 , and finally there are 10 different frequency bands, including 9 high-frequency components and 1 low-frequency components. The fusion rules of mean high-frequency components and low-frequency components are selected. In order to further illustrate the effectiveness of the proposed algorithm, the high-frequency part of the highest decomposition layer obtained by wavelet transform is fused by singular value contrast. 
Wang Jing, Zhang Guicang and Su Jinfeng

The level of wavelet decomposition is 3. Bior 2.4 is chosen as the mother wavelet and the neighborhood size is $3 \times 3$. Furthermore, the mean gradient averaging algorithm is used to fuse the low-frequency part of the highest decomposition layer and the high-frequency and low-frequency components of other decomposition layers.

Observing the experimental results, it is not difficult to find that two source images (a) and (b) are fused by w-

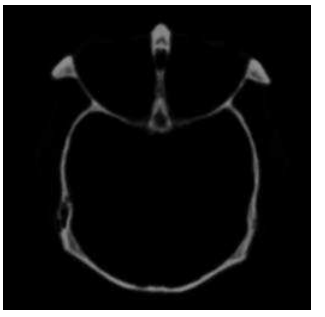

CT image (a)

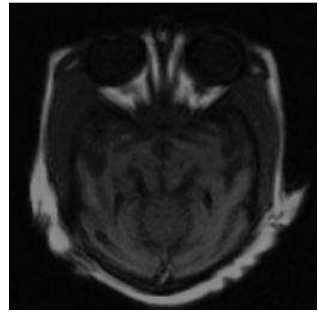

MRI image (b)
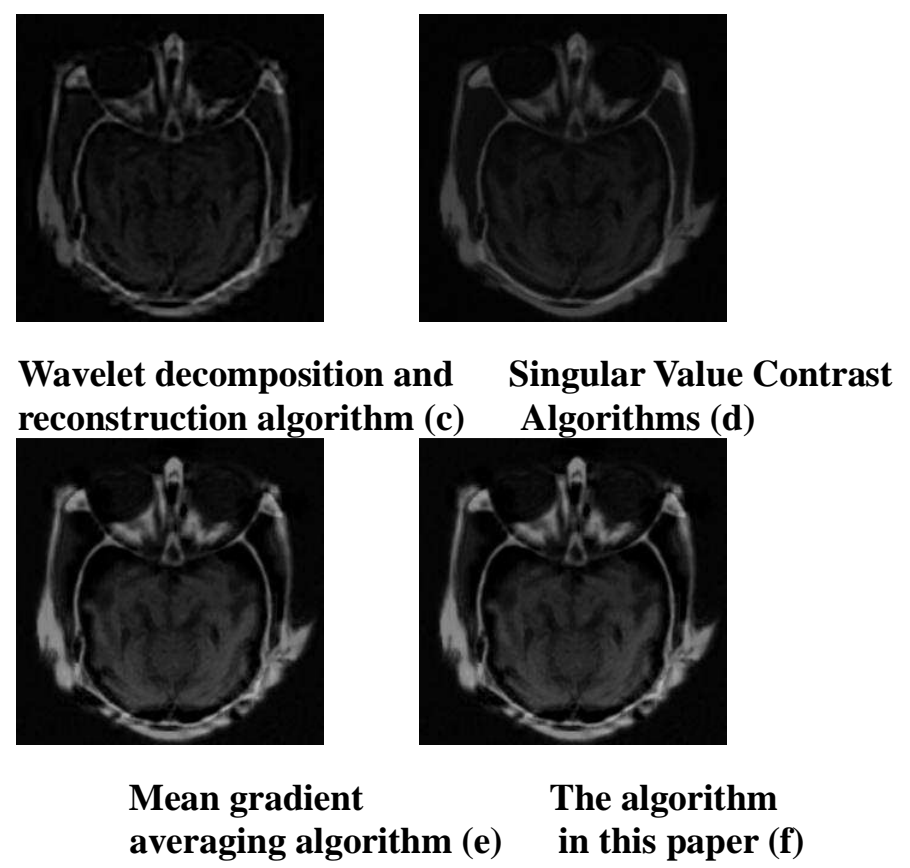

Figure 1: Comparison of different wavelet fusion algorithms with the proposed algorithm

Wavelet transform directly to get the image (c). There are too many details missing, which obviously contain irremovable noise and the contour is not clear. The image (d) is obtained by singular value contrast of the high frequency components of the highest decomposition layer after wavelet decomposition, although many noises are avoided and the clarity is improved, but the details information is also improved. The performance is not clear; the image (e) is obtained by averaging the low-frequency component of the 
Medical Image Fusion Combining Singular Value Decomposition and Mean Gradient

highest decomposition layer and the frequency-domain component of other layers after wavelet decomposition, and the clarity is greatly improved, but there is serious information loss in the image; the algorithm uses singular value contrast and average gradient mean to get the image (f) after wavelet decomposition, which basically eliminates the fusion process. The noise generated not only retains the texture information of the source image, but also enhances the clarity obviously. It shows that singular value decomposition and average gradient mean play an indispensable role in medical image fusion.

Table 1 presents the evaluation results of six different evaluation systems for different wavelet fusion algorithms. From the data in the table, it can be found that the evaluation criteria of the image obtained by the wavelet fusion are significantly lower than those of other algorithms; the image obtained by the singular value contrast algorithm has obvious improvement in information entropy ${ }^{[10]}$, average gradient ${ }^{[11]}$ and peak signal-to-noise ratio ${ }^{[12,17]}$, which shows that the singular value contrast algorithm has greatly improved the information extraction of medical image fusion; the image obtained by the average gradient mean algorithm Mean value ${ }^{[9]}$, information entropy and root mean square error ${ }^{[16]}$ have been greatly improved, which shows that the average gradient mean wavelet fusion algorithm can not only enhance the clarity of the fused image, but also avoid the noise. The wavelet fusion algorithm combining average gradient mean and singular value decomposition is the best except for the low peak signal-to-noise ratio, which shows that the average gradient is the best one. Degree mean and singular value decomposition play an indispensable role in medical image fusion.

In order to further illustrate the effectiveness of the proposed algorithm. The weighted averaging method, Laplacian pyramid algorithm and literature [4] are selected to compare the results of medical image fusion.In the e-

Table 1: Evaluation of different wavelet fusion methods and medical image fusion in this paper

\begin{tabular}{llccccc}
\hline Fusion algorithm & $\begin{array}{l}\text { mean } \\
\text { value }\end{array}$ & $\begin{array}{l}\text { standard } \\
\text { deviation }\end{array}$ & $\begin{array}{l}\text { Information } \\
\text { Entropy }\end{array}$ & $\begin{array}{l}\text { Average } \\
\text { gradient }\end{array}$ & $\begin{array}{l}\text { Peak } \\
\text { Signal-to- } \\
\text { Naion Dotin }\end{array}$ & $\begin{array}{l}\text { Root mean } \\
\text { square error }\end{array}$ \\
\hline Wavelet Fusion & 17.4120 & 18.7597 & 5.2015 & 2.0656 & 50.1317 & 0.1000 \\
$\begin{array}{l}\text { Singular value } \\
\text { contrasting }\end{array}$ & 17.4174 & 26.8144 & 5.4159 & 3.0241 & 53.8771 & 0.1060 \\
$\begin{array}{l}\text { Average gradient } \\
\text { averaging }\end{array}$ & 24.1286 & 30.7659 & 5.8137 & 3.6282 & 55.2916 & 0.1527 \\
$\begin{array}{l}\text { The algorithm in } \\
\text { this paper }\end{array}$ & 26.4904 & 32.8826 & 5.9859 & 4.0435 & 55.9575 & 0.1745 \\
\hline
\end{tabular}

Experiments, the optimal results obtained under the optimal environment are used as the comparison algorithms. The experimental results are shown in Figure 2. 
Wang Jing, Zhang Guicang and Su Jinfeng

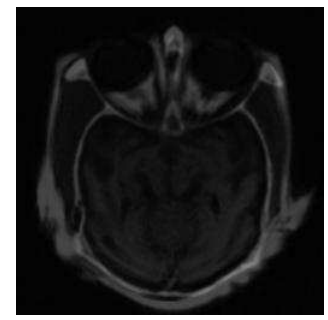

Weighted average $\operatorname{method}(\mathrm{g})$

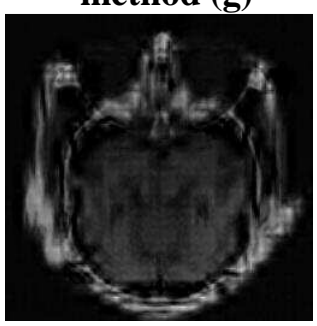

Literature [4]

Algorithms (i)

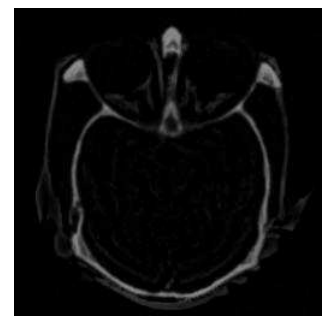

Laplacian pyramid algorithm (h)

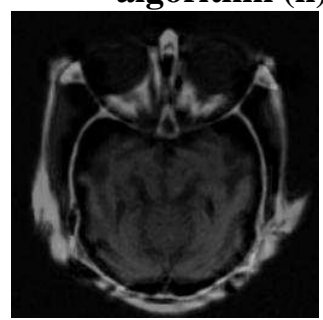

The algorithm in this paper (j)

Figure 2: Comparisons between the traditional algorithm and the algorithm presented in this paper

It is not difficult to find that the graph (g) obtained by weighted average method is not clear enough and the detail information is not fully expressed; the image (h) obtained by Laplace pyramid algorithm is slightly improved in clarity, but it produces a lot of noise and little detail information; the image (i) obtained by document [4] is not clear in detail, and the image is distorted, resulting in blurred image contour and loss of information. The loss is also very serious; the image (j) obtained by this algorithm is richer in detail than the image performance of the above algorithm, and the clarity is greatly improved, and the visual effect is also significantly better than the above algorithm, which shows that the algorithm in this paper has greatly improved in medical image fusion.

Table 2 gives the evaluation results of these algorithms. It can be seen that each index of the image obtained by weighted average method is significantly lower than that of other algorithms, which shows that the information contained in the fused image is very little; the image obtained by Laplace pyramid algorithm has a great improvement in standard deviation, information entropy, peak signal-to-noise ratio and root mean square error, which shows that the information and clarity of the image have been improved; although the image obtained by reference [4] algorithm is uniform. Value, standard deviation and root mean square error have been improved, but the information entropy and average gradient are significantly lower than the algorithm in this paper, which shows that the information of the image is greatly missing and the clarity is insufficient. The algorithm in this paper has the highest information entropy and average gradient, which means that the algorithm can transfer more useful information and edge information from the source 
Medical Image Fusion Combining Singular Value Decomposition and Mean Gradient image to the fused image, and the root mean square error is also the greatest. The result shows that the noise and artificial texture produced by the fusion image are very few, which can reduce the error of the doctor in diagnosis.

Table 2: Evaluation of medical image fusion based on different traditional fusion methods

\begin{tabular}{llcllcc}
\hline $\begin{array}{l}\text { Fusion } \\
\text { algorithm }\end{array}$ & $\begin{array}{l}\text { mean } \\
\text { value }\end{array}$ & $\begin{array}{l}\text { standard } \\
\text { deviation }\end{array}$ & $\begin{array}{l}\text { Information } \\
\text { Entropy }\end{array}$ & $\begin{array}{l}\text { Average } \\
\text { gradient }\end{array}$ & $\begin{array}{l}\text { Peak Signal- } \\
\text { to-Noise } \\
\text { Ratio }\end{array}$ & $\begin{array}{l}\text { Root mean } \\
\text { square error }\end{array}$ \\
\hline $\begin{array}{l}\text { weighted mean } \\
\begin{array}{l}\text { laplacian } \\
\text { pyramid }\end{array}\end{array}$ & 17.7264 & 18.8103 & 5.1515 & 2.0860 & 47.5599 & 0.0454 \\
$\begin{array}{l}\text { Literature [4] } \\
\begin{array}{l}\text { The algorithm } \\
\text { in this paper }\end{array}\end{array}$ & 22.76416 & 32.3110 & 5.8468 & 3.9101 & 55.8668 & 0.1684 \\
\hline
\end{tabular}

\section{Concluding remarks}

In order to solve the problem of image distortion and artificial texture caused by traditional fusion algorithms in the face of medical images, a medical image fusion algorithm combining singular value decomposition and average gradient is proposed, and two CT and MRI images are used for experiments. A large number of experiments show that the fusion rules of singular value decomposition and average gradient are used in the fusion phase, which not only stabilizes the pixels, but also enriches the details of the fused image, and solves the problems of traditional methods. Although the proposed algorithm for medical image fusion has achieved good results, how to apply this method to other modal medical image fusion is the focus of future research.

\section{REFERENCES}

1. Liu Shuaiqi, Zheng Wei, Zhao Jie and Hu Shaohai, Analysis and Application of Digital Image Fusion Algorithms, 1st Edition. Beijing: Machinery Industry Press, (2018) 138-203.

2. Xiao Jinsheng, Rao Tianyu, Jiaxi, Song Jinzhong and Yi Benshun, Laplacian pyramid image fusion algorithm based on graph cutting, Optoelectronic Laser, 25(7) (2014) 1416-1424.

3. Quan Yanan, Brijing and Wu Wenbo, Improved threshold weighted average HSV and wavelet transform image fusion, Journal of Liaoning University of Engineering and Technology, 35 (1) (2016) 65-69.

4. Zhao Qing, He Jianhua and Wen Peng, Image fusion method based on average gradient and directional contrast, Computer Engineering and Application, 48(24) (2012) 165-168.

5. Wang Xiaobo and Liu Bin, Multi-focus image fusion based on multi-resolution singular value decomposition, Journal of Quantum Electronics, 31(3) (2014) 257-263.

6. Chen Guangqiu, Gaoyin Han, Duan Jin and Han Zeyu, PCNN infrared and visible 
Wang Jing, Zhang Guicang and Su Jinfeng

image fusion based on singular value decomposition, LCD and display, 30(1) (2015) 127-136.

7. J.Liang, Y.He, D.Liu and X.Zeng, Image fusion using higher order singular value decomposition, IEEE Transactions on Image Processing, 21(5) (2012) 2898-909.

8. Boyang Cheng, Longxu Jin and Guoning Li, A novel fusion framework of visible light and infrared images based on singular value decomposition and adaptive DUAL-PCNN in NSST domain, Infrared Physics \& Technology, 91 (2018) 153-163.

9. Daihua, Matrix Theory, 3rd Edition. Beijing: Science Press, (2001) 117-140.

10. Zhang Xiaoli, Li Xiongfei and Li Jun, Relevance analysis and performance report of fusion image quality evaluation index, Journal of Automation, 40(2) (2014) 306-312.

11. Zhiping $\mathrm{Xu}$, Medical image fusion using multi-level local extrema, Information Fusion, 19(1) (2014) 38-48.

12. Xinzheng $\mathrm{Xu}$, Dong Shan, Guanying Wang and Xiangying Jiang, Multimodal medical image fusion using PCNN optimized by the QPSO algorithm, Applied Soft Computing, 46 (2016) 588-595.

13. Rajiv Singh and Ashish Khare, Fusion of multimodal medical images using Daubechies complex wavelet transform - A multire solution approach, Information Fusion, 19 (2014) 49-60.

14. V.Bhavana and H.K.Krishnappa, Multi-modality medical image fusion using discrete wavelet transform, Procedia Computer Science, 70 (2015) 625-631.

15. Reena Benjamin and T.Jayasree, Improved medical image fusion based on cascaded PCA and shift invariant wavelet transforms, International Journal of Computer Assisted Radiology and Surgery, 13(2) (2018) 229-240.

16. Baohua Zhang, Chuanting Zhang, Jianshuai Wu and He Liu, A medical image fusion method based on energy classification of BEMD components, Optik International Journal for Light and Electron Optics, 125(1) (2014) 146-153.

17. Hong-jie Li, Gui-Cang Zhang and Zong-yuan Zhu, Color Image Segmentation Based on PCNN, Journal of Mathematics and Informatics, (2018) 41-53.

18. Liang-liang Wang, Gui-cang Zhang and Wen-xiao Jia, Adaptive image fusion method based on non-subsampled contourlet wavelet transform, Journal of Mathematics and Informatics, (2018) 1-10.

19. Si-qi Han, Joint up-sampling high dynamic range images with a guidance image, Journal of Mathematics and Informatics, (2019) 59-71. 